\title{
Surgical success and lateral incomitance following three-muscle surgery for large-angle horizontal strabismus
}

\author{
Diana L. Cifuentes, MDa , Stacy L. Pineles, MDa , Joseph L. Demer, MD, PhD ${ }^{a, b, c, d, e}$, and \\ Federico G. Velez, MD ${ }^{a, f, g}$ \\ aDepartment of Ophthalmology, Jules Stein Eye Institute, University of California Los Angeles \\ ${ }^{b}$ Department of Mechanical Engineering, University of California Los Angeles \\ 'Biomedical Engineering Interdepartmental Program, University of California Los Angeles \\ dNeuroscience Interdepartmental Program, University of California Los Angeles \\ eDepartment of Neurology, University of California Los Angeles \\ fDepartment of Ophthalmology, Doheny Eye Institute, University of California Los Angeles \\ gDepartment of Surgery, Olive View-UCLA Medical Center, Sylmar, California
}

\begin{abstract}
Purpose-To evaluate motor alignment, motility, and sensorial outcomes of simultaneous threemuscle surgery for large-angle horizontal strabismus, with special attention to lateral incomitance and long-term success.
\end{abstract}

\begin{abstract}
Methods-The medical records of consecutive patients with large-angle deviations ( $230^{\Delta}$ ) who underwent simultaneous surgery on three horizontal muscles to correct esotropia or exotropia were reviewed retrospectively. Successful motor alignment was defined as residual deviation of $\leq 0^{\Delta}$ and consecutive deviation in primary position of $4^{\Delta}$, with no induced lateral incomitance $>5^{\Delta}$ between lateral gazes. Sensory success was defined as an improvement in stereopsis of $\geq 2$ octaves. Surgical procedures included a combination of recessions and resections/plications depending on surgeon preference. The majority of cases were two-muscle recessions combined with one-muscle resection or plication.
\end{abstract}

Results-A total of 19 patients with exotropia and 9 patients with esotropia were included. In the esotropic group, the mean age at surgery was $48 \pm 15$ years and the mean preoperative deviation improved from $45.6^{\Delta} \pm 11.9^{\Delta}$ to $1.5^{\Delta} \pm 1.6^{\Delta}(P<0.001)$; there was no undercorrection, recurrence or overcorrection. In the exotropic group, the mean age at surgery was $44 \pm 25$ years, and the mean preoperative deviation improved from $44.1^{\Delta} \pm 8.7^{\Delta}$ to $5.8^{\Delta} \pm 9.6^{\Delta}(P<0.001)$, recurrence occurred in $2(22 \%)$. Overall motor success at distance was $85 \%$, with $1(3.8 \%)$ unsuccessful patient due to induced incomitance. Sensory success was $44 \%$ for esotropia and $31 \%$ for exotropia. No patient lost stereopsis.

Correspondence: Federico G Velez, 100 Stein Plaza, David Geffen School of Medicine at UCLA, Los Angeles, CA 90095-7002 (velez@jsei.ucla.edu). 
Conclusions-Patients undergoing three-muscle surgery for horizontal strabismus had good motor outcomes with low incidence of induced lateral incomitance. Motor and sensorial outcomes for esotropia were very stable.

The goal of surgical treatment of large-angle strabismus is to achieve a satisfactory motor alignment in primary position without limiting ocular rotations or inducing lateral incomitance. Some investigators have defined large-angle esotropia as a deviation of $30^{\Delta}$ and a large-angle exotropia as a deviation $>35^{\Delta}$; others consider $>40^{\Delta}$ to be large. ${ }^{1-12}$ There is currently no consensus on how many muscles to operate on for large-angle strabismus. ${ }^{10-11}$ However, it is generally agreed that an important consideration when performing asymmetric surgery is avoidance of induction of lateral incomitance. ${ }^{10,13-14}$ Those who prefer to operate on three or four horizontal rectus muscle surgery for esotropia argue that this approach helps to diminish the chances of convergence impairment and incomitance, which can result from excessive medial rectus recession and may contribute to a late exotropic drift in patients with esotropia. ${ }^{6}$ Previous studies have reported that overall success of three-muscle surgery for large-angle horizontal strabismus ranges from $42 \%$ to $83 \%$. 5-6,9-10,15-17 Limited information is available regarding lateral incomitance. The purpose of this study was to evaluate alignment, motility, and binocularity outcomes of threehorizontal-muscle surgery for large-angle horizontal strabismus, with special attention to induced lateral incomitance and long-term success.

\section{Subjects and Methods}

This study was approved by the University of California Institutional Review Board. The medical records of all consecutive patients with horizontal strabismus, exotropia, and esotropia measuring $230^{\Delta}$ who underwent simultaneous surgery on three horizontal rectus muscles at the Stein Eye Institute, University of California Los Angeles between 2008 and 2016 were reviewed retrospectively. Information registered at the last follow-up visit or before reoperation was used for analysis. Patients were included if they completed at least 6 weeks of postoperative follow-up. Exclusion criteria included strabismus secondary to paresis, paralysis, restriction or misinnervation, history of amblyopia, nystagmus, neurological disease, and previous strabismus surgery.

Motor alignment and sensory function were evaluated with patients wearing the appropriate optical correction to obtain best visual acuity. Ocular alignment was measured pre- and postoperatively using the alternate prism cover test in the six cardinal diagnostic positions of gaze at 12 feet and at near using an accommodative target at 14 inches. ${ }^{18}$ In patients with poor visual acuity, motor alignment examination was assessed with the Krimsky test. ${ }^{18}$ Lateral gazes incomitance was calculated in prism diopters as the difference between the angle of deviation measured in lateral gazes. Ocular rotations were measured using a scale ranging between 0 and $\pm 4 .^{18}$

Sensory function was measured at near using the Titmus Fly stereoacuity test (Titmus Optical Co Inc, Petersburg, VA). Patients were classified into one of four groups for stereopsis outcomes as follows: bifoveal fusion, 40 arcsec; peripheral fusion, 50-300 arcsec; gross stereopsis, >300-3552 arcsec; and no stereopsis, >3552 arcsec. Stereopsis outcomes 
were assessed as a continuous variable by taking the natural $\log$ of the value of stereopsis, assigning 10,000 sec to "no stereopsis."

Intraoperative forced duction testing (FDT) ${ }^{19}$ was performed at the beginning of the surgery. Surgical procedures included a combination of recessions and resections or recessions and plications, using standard surgical techniques. ${ }^{20,21}$ Surgical strategy was determined by the results of FDT $^{19}$; recessions were performed whenever the FDT revealed a tight muscle and also were the preferred bilateral procedure when FDT was negative.

Surgeries were performed by three surgeons using a standard surgical technique (same dose of surgery was done in the same muscle of each eye), except that one surgeon (JLD) preferred plications to resections. Two surgeons (SLP, FGV) followed an algorithm to calculate the amount of surgery by dividing the total distance angle of deviation in the primary position by 2 then determining the amount of recession plus resection/plication for that "half angle" in one eye and performing the same amount of surgery on the same muscle on the fellow eye; in most cases a recession was performed in the fellow eye, except for 2 patients in the esotropic group. The eye that had two-muscle surgery was always the eye with worse visual acuity in the cases where visual acuity differed between eyes. The third surgeon (JLD) followed an algorithm based on a total amount of surgery of 18-19 mm total divided among the three operated muscles, with the same muscle operated on each eye having the same dose of recession. Whenever possible, adjustable sutures were used, using the bow tie technique. ${ }^{22}$

All patients were examined within 3 hours after surgery; postoperative adjustment was performed in the recovery room. The target angle for adjustment was orthotropia at distance for esotropia and a small esotropia $\left(<4^{\Delta}\right)$ for exotropia, measured with the patient wearing the appropriate optical correction fixing on a target at a distance of 20 feet. All patients were examined within the first 2 weeks of and at variable time points thereafter for at least 6 weeks.

Surgical success was calculated using the motor-alignment/sensory definition in Table 1 . Phorias were considered a successful result. The long-term success was assessed with the recurrence rate, defined as percentage of patients with undercorrection of $>10^{\Delta}$ at 6 months' follow-up. Means were compared using the $t$ test, with $P$ values $<0.05$ considered statistically significant.

\section{Results}

A total of 28 patients (16 males) were included, 9 with esotropia and 19 with exotropia. Neither age at presentation ( $21 \pm 22$ years for esotropia [range, $1-56$ years] and $30 \pm 28.2$ for exotropia [range, 3-82 years]) nor age at surgery (48 \pm 15 years for esotropia [range, 3379 years] and $44 \pm 25$ years for exotropia [range 3-82 years]) differed significantly between groups. The majority of patients with esotropia were female $(67 \%)$; the majority with exotropia, males (68\%). All 9 patients with esotropia were adults when surgery was performed; 5 were presumed to have infantile esotropia, according to patient information, and 4 had acquired esotropia. The esotropia was constant in all patients. Of the 19 exotropia 
patients, 9 (48\%) had an intermittent poorly controlled deviation, $17(89 \%)$ had a basic type, and $2(11 \%)$ had a divergence excess type. Motor-alignment characteristics are shown in Table 2 .

Three patients underwent simultaneous vertical muscle surgery; 1 esotropic patient underwent a recession of the left superior rectus for a hypertropia, 1 exotropic patient underwent bilateral inferior oblique recessions, and 1 exotropic patient underwent superior rectus recession for a hypertropia.

All esotropic patients underwent recession of one medial rectus on adjustable suture; 5 patients had simultaneous lateral rectus muscle resection or plication on adjustable suture. Three patients (33\%) required postoperative adjustment for immediate overcorrection of $13^{\Delta}$ $\pm 7^{\Delta}$, with a post-adjustment esotropia of $1^{\Delta}$. Fifteen adult exotropic patients underwent adjustable suture surgery. All had one adjustable lateral rectus muscle recession and 4 patients had simultaneous adjustable medial rectus muscle resection or plication. One exotropic patient underwent postoperative adjustment with a final postoperative adjustment target of $4^{\Delta}$. All of the patients underwent a two-muscle recession plus a resection/plication of one muscle, except for 2 esotropic patients. These 2 underwent one-muscle recession, combined with two-muscle resection in one case and two-muscle plication the other; these patients had a negative FDT. No complications were presented intra- or postoperatively in either group.

Preoperative duction examination revealed limitation to abduction in $67 \%$ of esotropic patients (mean, $-1.5 \pm 0.5$ ) and limitation to adduction in $16 \%$ of exotropic patients (mean, $-1.2 \pm 0.2)$.

Mean postoperative follow-up was $12.5 \pm 1$ months (range, 6 weeks to 57 months). Overall motor success was $89 \%$ at near and $85 \%$ at distance at final follow-up or before a reoperation; 9 of 9 patients with esotropia achieved motor success at distance and near; of these, 5 had a preoperative deviation of $30^{\Delta}-49^{\Delta}$ and 4 patients had preoperative deviations of $250^{\Delta}$. For the exotropia group, 16 of $19(84 \%)$ patients had a successful surgical outcome at near; 14 of $17(82 \%)$, at distance. Thirteen patients had a preoperative deviation of 30$49 \mathrm{D}$ and $6 \geq 50 \mathrm{D}$. The 3 unsuccessful patients from this group were undercorrected at distance and near, 1 with induced incomitance; all 3 had a preoperative deviation between $30-49 \searrow \mathrm{D}$ and none $250 \mathrm{D}$. Thirteen patients from exotropia group had a deviation of $30^{\Delta}$ $-49^{\Delta}$ and $6^{\Delta}$ to $\geq 50^{\Delta}$. Our success rate for preoperative deviations $\geq 50^{\Delta}$ was $100 \%(5 / 5)$ regardless of the type of deviation and $75 \%$ (9/12) in the exotropia group for deviations of $30^{\Delta}-49^{\Delta}$. Mean preoperative esotropic deviation improved from $45.6^{\Delta} \pm 11.9^{\Delta}\left(30^{\Delta}-70^{\Delta}\right)$ to $1.5 \pm 1.6^{\Delta}\left(0^{\Delta}-4^{\Delta}\right)$ at distance $(P<0.0001)$ and from $43.7^{\Delta} \pm 20.1^{\Delta}$ (range, $\left.14^{\Delta}-70^{\Delta}\right)$ to 0.4 $\pm 0.8^{\Delta}$ (range, $\left.0^{\Delta-2} 2^{\Delta}\right)$ at near $(P<0.0001)$. Mean preoperative exotropic deviation improved from $44.1^{\Delta} \pm 8.7^{\Delta}$ (range, $25^{\Delta}-63^{\Delta}$ ) to $5.8^{\Delta} \pm 9.6^{\Delta}$ (range, $\left.0^{\Delta}-35^{\Delta}\right)$ at distance $(P<0.0001)$ and from $40.8^{\Delta} \pm 15.7^{\Delta}$ (range, $\left.0^{\Delta}-60^{\Delta}\right)$ to $5.0 \pm 11.2^{\Delta}$ (range, $\left.0^{\Delta}-45^{\Delta}\right)$ at near $(P<0.0001)$.

Three-muscle strabismus surgery resolved the preoperative lateral incomitance in 6 of 7 patients (86\%) by changing the lateral comitance in those patients who had preoperative lateral incomitance; 20 patients did not have preoperative incomitance, and one 3-year-old 
exotropic patient motor alignment was assessed with the Krimsky test and pre- and postoperative lateral incomitance could not be evaluated. Rest of the patients were evaluated with cover testing. Surgery induced lateral incomitance in 1 of $26(3.8 \%), 2$ patients from exotropia group were not measured in lateral gazes postoperatively. One patient with esotropia had preoperative incomitance that completely resolved after surgery, the other 6 patients belonged to the exotropia group, and only 1 patient remained incomitant postoperatively. One patient from the exotropia group had induced incomitance, none from the esotropia group. For patients with $>6$ months' follow-up $(n=14)$, there was recurrence in 2 of 9 exotropic patients (22\%) but in none with esotropia.

Sensory evaluation is presented in Table 3. Of those tested for stereopsis, sensory success was achieved in 4 of 9 esotropic patients (44\%) and 5 of 16 exotropic patients (31\%). Three patients were too young to evaluate stereopsis. In the esotropic group only 2 patients had preoperative stereopsis: one with acquired esotropia improved from $100 \operatorname{arcsec}$ to $40 \mathrm{arcsec}$; the other, with presumably infantile esotropia, remained stable with 3552 arcsec. These 2 patients had a constant esotropia at distance with an intermittent deviation at near. In the exotropia group, 2 patients measured negative preoperative stereopsis: one remained stable, and the other improved to $3552 \mathrm{arcsec}$. The mean log preoperative stereopsis in esotropic patients $(8183.5 \pm 3706.2 \mathrm{arcsec})$ improved significantly $(2116 \pm 3283.7 \operatorname{arcsec}[P \leq 0.05])$; this was not the case in the exotropic group $(1874.8 \pm 3369.3$ to $1214 \pm 2616.2 \operatorname{arcsec}[P>$ 0.05]). No patient lost stereopsis.

\section{Discussion}

Our findings reveal that three-muscle surgery can result in good motor alignment for patients with large-angle horizontal strabismus. The outcomes were successful in all of our esotropic patients in terms of motor alignment, with no induced incomitance and no recurrences; success was achieved in $82 \%$ of cases (14/17) in the exotropia group, with 6\% (1/17) induced incomitance and a $22 \%$ recurrence rate (2/9).

Previous reports following three-muscle surgery for patients with large-angle strabismus have shown undercorrection rates of $25 \%-58 \%$ in exotropic patients and of $2 \%-20 \%$ in esotropic patients. Overcorrection rates reported for esotropic patients are 5\%-60\% and $0 \%$ $-6.7 \%$ for exotropic patients after three-muscle surgery. $6,8-11,15-17,23$ In our series, undercorrections were seen in 3 exotropic patients (16\%) compared to no undercorrections in esotropic patients; there were no overcorrections. Of the 3 undercorrected exotropic patients, 2 underwent adjustable suture surgery: immediate postoperative alignment was orthotropia in one patient and $10^{\Delta}$ of esotropia in the other, but neither patient was adjusted. Previous study by Pineles and colleagues ${ }^{24}$ demonstrated that the amount of overcorrection on postoperative day 1 was not statistically significant to predict long-term alignment.

Graeber and Hunter ${ }^{14}$ reported that asymmetric surgery induces more lateral incomitance than symmetric surgery $(10.8 \%$ vs $1.2 \%)$. When patients presented with preoperative lateral incomitance, asymmetric surgery tended to correct the lateral differences more than symmetric surgery ( $41 \%$ vs $5 \%$ ). In their series, three-muscle strabismus surgery-induced lateral incomitance was $12.5 \%$ (2/16) while in 33\% (1/3) lateral incomitance was eliminated. 
In our study, three-muscle surgery eliminated the preoperative lateral incomitance in $86 \%$ of patients $(6 / 7)$, while $<4 \%(1 / 26)$ had induced lateral incomitance. This restoration of comitance could be explained by the fact that binocular vision was recovered following surgery, leading to a recovery of fusion in side gazes; additionally, surgery might have been planned in order to correct the incomitance by operating on the muscles responsible of the deviation in a particular horizontal gaze. Of note, the three-muscle surgery changed the lateral comitance-in the majority of our cases with preoperative lateral incomitance the change was beneficial, lessening the individual patients' lateral incomitance. Neither of the patients with preoperative lateral incomitance experienced a worsening of the incomitance. The single patient that did not have a lessening of incomitance had no change in the amount of incomitance.

Patients with preoperative diplopia from the esotropia group did not have diplopia postoperatively. In the exotropia group, of 8 patients with preoperative diplopia, only 1 from the constant exotropia group remained diplopia free in side gazes, and 1 patient had intermittent postoperative diplopia de novo. This patient had $2^{\Delta}$ of esotropia postoperatively, and it is likely that the consecutive esotropia caused this intermittent diplopia. When performing three-muscle surgery most authors recommend recessing two muscles and resecting one muscle in the nondominat eye. ${ }^{6-7,16}$

In patients with large-angle congenital esotropia, Scott and colleagues ${ }^{11}$ used two surgical approaches: (1) the uniform approach, consisting of surgery on two muscles either bilaterally or monocularly; and (2) the selective approach, consisting of surgery on three or four horizontal muscles. A good result was achieved in $37.3 \%$ of cases in the uniform surgery group, with a mean preoperative deviation of $55^{\Delta}$, and $64.6 \%$ in the selective group, with a mean preoperative deviation of $65^{\Delta}{ }^{8}$ Lau and colleagues ${ }^{16}$ followed an algorithm of bilateral lateral rectus muscle recessions of $9.0 \mathrm{~mm}$ for $50^{\Delta}$ of exotropia combined with 1 $\mathrm{mm}$ resection of a medial rectus muscle for each additional $5^{\Delta}$ of exotropia over $50^{\Delta}$ and reported a success rate of $75 \% .{ }^{16}$ In our study the success for horizontal deviations $250^{\Delta}$ was $100 \%$.

For esotropia we had a dose-response correlation of $4.7^{\Delta}$ per mm, using the first surgical approach described above. Despite the common approach of using half the deviation as the target angle for each eye and performing recession-resection procedures bilaterally for largeangle deviations, our approach for three-muscle surgery, using only a unilateral recessionresection for half of the deviation combined with a single recession in the fellow eye suggests that less surgery suffices for correction. In our patients with exotropia, we found that regardless of the surgical dose, patients had a good response at final follow-up, suggesting that the effect of surgery was more closely related to the preoperative deviation than to the amount of surgical change per mm, as mentioned by Archer and Kushner. ${ }^{25,26}$

More exotropic patients had stable stereopsis (55\%) than those patients in the esotropia group (22\%); this may be because baseline stereopsis was more frequently present in this group (exotropia $88 \%$ vs esotropia $22 \%$ ) with $48 \%$ of the exotropia group having intermittent deviations compared to the constant esotropia in $100 \%$ of the esotropic group. 
Kushner and colleagues found for longstanding strabismus that regardless of the duration and type of deviation patients can develop binocularity after surgery. ${ }^{27}$

Our study has several limitations. This is a retrospective review of records, with a relatively small number of patients and short follow-up period. This study lacks a comparison of surgeries in two, three, and four muscles; further studies are needed to compare techniques. Some pre- and postoperative motor and sensorial data were not available; more than one examiner was involved, and this can affect measurements of, for example, lateral incomitance. Despite the standard surgical techniques, three surgeons performed all procedures, and some variability in technique may have affected the results. Two muscletightening procedures were used, and although the procedures have been reported to be equivalent, the long-term effect of those procedures or their effect in lateral incomitance may differ. ${ }^{28}$ Despite these limitations, our study indicates that three-muscle surgery effectively treats large-angle strabismus, with a low rate of induced lateral incomitance.

\section{Acknowledgments}

Financial support: NIH/NEI-K23EY021762, Research to Prevent Blindness, Walt and Lily Disney Award (SLP); NEI-EY008313, Research to Prevent Blindness (JLD); Research to Prevent Blindness (FGV).

\section{References}

1. Costenbader FD. Infantile esotropia. Trans Am Ophthalmol Soc 1961;59:397-429. [PubMed: 13881627]

2. Hess JB, Calhoun JH. A new rationale for the management of large angle esotropia. J Pediatr Ophthalmol Strabismus 1979;16:345-8. [PubMed: 521874]

3. Schiavi C, Bolzani R, Benassi MG, Bellusci C, Campos EC. Visual recognition time in strabismus: small-angle versus large-angle deviation. Eur J Ophthalmol 2004;14:200-205. [PubMed: 15206644]

4. Assaf AA. Large bimedial rectus recession ( $6.5 \mathrm{~mm}$ or more) in the management of large-angle esotropia. Strabismus 1997;5:59-66. [PubMed: 21314395]

5. Chatzistefanou KI, Ladas ID, Droutsas KD, Koutsandrea C, Chimonidou E. Three horizontal muscle surgery for large angle infantile or presumed infantile esotropia long-term motor outcomes. JAMA Ophthalmol 2013;131:1041-8. [PubMed: 23699727]

6. Forrest MP, Finnigan S, Finnigan BAS, Gole GA. Three horizontal muscle squint surgery for large angle infantile esotropia. Clin Exp Ophthalmol 2003;31:509-16. [PubMed: 14641159]

7. Livir-Rallatos G, Gunton KB, Calhoun JH. Surgical results in large angle exotropia. J AAPOS 2002;6:77-80. [PubMed: 11997802]

8. Currie ZI, Shipman T, Burke JP. Surgical correction of large angle exotropia in adults. Eye 2003; 17 : 334-9. [PubMed: 12724695]

9. Thomas S, Guha S. Large-angle strabismus: can a single surgical procedure achieve a successful outcome? Strabismus 2010;18:129-36. [PubMed: 21091333]

10. Millan T, de Carvalho KM, Minguini N. Results of monocular surgery under peribulbar anesthesia for large angle horizontal strabismus. Clinics 2009;64:303-8. [PubMed: 19488586]

11. Scott WE, Reese PD, Hirsh CR, Flabetich CA. Surgery for large-angle congenital esotropia two vs three and four horizontal muscles. Arch Ophthalmol 1986;104:374-7. [PubMed: 3954636]

12. Camuglia JE, Walsh MJ, Gole GA. Three horizontal muscle surgery for large-angle infantile esotropia: validation of a table of amounts of surgery. Eye 2011;25:1435-41. [PubMed: 21818127]

13. Deacon BS, Fray KJ, Grigorian AP et al. Unilateral strabismus surgery in patients with exotropia results in postoperative lateral incomitance. J AAPOS 2014;18:572-5. [PubMed: 25498465]

14. Graeber CP, Hunter DG. Changes in lateral comitance after asymmetric horizontal strabismus surgery. JAMA Ophthalmol 2015;133:1241-6. [PubMed: 26291652] 
15. Chen JH, Morrison DG, Donahue SP. Three and four horizontal muscle surgery for large angle exotropia. J Pediatr Ophthalmol Strabismus 2015;52:305-10. [PubMed: 26098544]

16. Lau FHS, Fan DSP, Yip WWK, Yu CBO, Lam DSC. Surgical outcome of single staged three horizontal muscles squint surgery for extra-large angle exotropia. Eye 2010;24:1171-6. [PubMed: 20139912]

17. Li JH, Zhang LJ. Three-muscle surgery for very large-angle constant exotropia. J AAPOS 2013;17:578-81. [PubMed: 24210348]

18. Mehta A Chief complaint, history and physical examination In: Rosenbaum AL, Santiago AP, eds. Clinical strabismus management: principles and surgical techniques. Philadelphia, PA: W. B. Saunders Co; 1999:3-21.

19. Santiago AV, Rosenbaum AL. Tests of muscle function In: Rosenbaum AL, Santiago AP, eds. Clinical strabismus management: principles and surgical techniques. Philadelphia, PA: W. B. Saunders Co.; 1999:37-51.

20. Velez FG, Demer JL, Pihlblad MS, Pineles SL. Rectus muscle plication using an adjustable suture technique. J AAPOS 2013;17:480-83. [PubMed: 24160967]

21. Oltra EZ, Pineles SL, Demer JL, Quan AV, Velez FG. The effect of rectus muscle recession, resection and plication on anterior segment circulation in humans. Br J Ophthalmol 2015;99:55660. [PubMed: 25342275]

22. Siegel LM, Lozano MJ, Santiago AP, Rosenbaum AL. Adjustable and nonadjustable recession and resection techniques In: Rosenbaum AL, Santiago AP, eds. Clinical strabismus management: principles and surgical techniques. Philadelphia PA: W. B. Saunders Co;1999:435-48.

23. Yang Chen J, Shen T, et al. Single stage surgical outcomes for large angle intermittent exotropia. PLoS One 2016;11:e0150508. doi: 10.1371/journal.pone.0150508.

24. Pineles SL, Deitz LW and Velez FG. Postoperative outcomes of patients initially overcorrected for intermittent exotropia. J AAPOS 2011;15:527-31. [PubMed: 22153394]

25. Archer SM. The effect of medial versus lateral rectus muscle surgery on distance-near incomitance. J AAPOS 2009;13:20-26. [PubMed: 19131260]

26. Kushner BJ, Fisher MR, Lucchese NJ, Morton GV. factors influencing response to strabismus surgery. Arch Ophthalmol 1993;111:75-9. [PubMed: 8424728]

27. Kushner BJ, Morton GV. Postoperative binocularity in adults with longstanding strabismus. Ophthalmology 1992;99:316-19. [PubMed: 1565442]

28. Chaudhuri Z, Demer JL. Outcomes following rectus muscle plication a potentially reversible, vessel-sparing alternative to resection. JAMA Ophthalmol 2014;132:579-85. [PubMed: 24676145] 
Table 1.

Motor-alignment/sensory definition

\begin{tabular}{|c|c|}
\hline Criteria & Exotropia-esotropia \\
\hline \multicolumn{2}{|c|}{ Motor alignment success } \\
\hline at distance & $\begin{array}{l}\triangle 10 \mathrm{PD} \text { residual deviation and } \leq 4 \mathrm{PD} \text { of consecutive deviation in PP with no induced lateral incomitance } \geq 5 \text { PD } \\
\text { between lateral gazes }\end{array}$ \\
\hline at near & $\leq 10 \mathrm{PD}$ residual deviation and $\leq 4 \mathrm{PD}$ consecutive deviation in PP \\
\hline Sensory success & Improvement in stereopsis of $\geq 2$ octaves \\
\hline Overcorrection & $>4 \mathrm{PD}$ consecutive deviation for distance and near in PP \\
\hline Undercorrection & $>10 \mathrm{PD}$ residual deviation for distance and near in PP \\
\hline
\end{tabular}

$P D$, prism diopter; $P P$, primary position. 
Table 2.

Motor-alignment characteristics

\begin{tabular}{|c|c|c|c|c|}
\hline \multirow[t]{2}{*}{ Characteristic } & \multicolumn{2}{|r|}{ Preoperative } & \multicolumn{2}{|r|}{ Postoperative } \\
\hline & Esotropia & Exotropia & Esotropia & Exotropia \\
\hline Diplopia & $\mathrm{n}=5(55 \%)$ & $\mathrm{n}=8$ (5 with IXT and 3 with CXT) & $\mathrm{n}=0$ & $\mathrm{n}=4$ (all IXT, 1 de novo) \\
\hline Incomitance & $\mathrm{n}=1(11 \%)$ & $\mathrm{n}=6(32 \%)$ & $\mathrm{n}=0$ & $\begin{array}{l}\mathrm{n}=2(11 \%) \\
\text { Residual: } \mathrm{n}=1(14 \%) \\
\text { Induced: } \mathrm{n}=1(5.8 \%)\end{array}$ \\
\hline $\begin{array}{l}\text { Mean angle incomitance |right gaze } \\
\text { - left gaze| }\end{array}$ & $\mathrm{n}=1 ; 10 \mathrm{PD}$ & $\mathrm{n}=6 ;$ mean, $12.3 \pm 4.5 \mathrm{PD}($ range, $7-20 \mathrm{PD})$ & $\mathrm{n}=0$ & $\begin{array}{l}\text { Residual: } 12 \mathrm{PD} ; \mathrm{n}=1 \\
\text { Induced: } 6 \mathrm{PD} ; \mathrm{n}=1\end{array}$ \\
\hline
\end{tabular}

$I X T$, intermittent exotropia. 
Table 3.

Preoperative and postoperative stereopsis groups

\begin{tabular}{lccccccccc}
\hline \multicolumn{1}{c}{ Stereopsis status } & \multicolumn{2}{c}{$\begin{array}{c}\text { No } \\
\text { stereopsis }\end{array}$} & \multicolumn{2}{c}{ Gross } & \multicolumn{2}{c}{$\begin{array}{c}\text { Peripheral } \\
\text { stereopsis }\end{array}$} & \multicolumn{2}{c}{$\begin{array}{c}\text { Bifoveal } \\
\text { fusion }\end{array}$} \\
& ET & XT & ET & XT & ET & XT & ET & XT \\
\hline Preoperative, no. patients & 7 & 2 & 1 & 6 & 1 & 6 & 0 & 2 \\
Postoperative, no. patients & 1 & 1 & 4 & 5 & 2 & 7 & 2 & 3 \\
\hline ET, esotropia; $X T$, exotropia. & & & & & & & & \\
\end{tabular}

